\title{
A Fiber-Optic Icing Sensor with Multi-Light Paths
}

\author{
Kan Lu and Feng Chen ${ }^{2, *}$ \\ Information Science and Technology, University of Science and Technology of China, China \\ * Corresponding author
}

\begin{abstract}
Fiber-optic icing sensor is a kind of light-intensitymodulation sensor. It can indirectly measure the ice thickness based on light intensity. To increase measuring scope of the reflective fiber-optic icing sensor, a fiber-optic icing sensor with multi-light paths is presented. , a set of fiber array is introduced to measure the transmission intensity of the light for the reflective fiber-optic icing sensor. The Support Vector Machine Regression Algorithm is utilized to fuse the transmission intensity and reflected intensity for estimate the ice thickness. The experimental results show that this proposed method can improve the measurement accuracy and range of the fiber-optic icing sensor. The anti-interference capability of the fiber-optic icing sensor is enhanced simultaneously.
\end{abstract}

Keywords-multi-light paths; support vector machine regression; fiber-optic icing sensor

\section{INTRODUCTION}

The phenomena of road icing occur from November to April in most parts of China. Icy roads easily cause the traffic accidents and property loss. In early 2008, a number of provinces in southern China have serious snow disaster, which resulted in large region paralysis of traffic. Nowadays, there are popular ice measurement methods such as differential pressure, vibrating-tube, and capacitive method. However, it is highly difficult for these methods to meet the demands of both small volume and high precision. In recent years, there have been a lot of research results on fiber-optic icing sensor, which has advantages of sensitivity and volume. There are two main kinds of fiber-optic icing sensors: the reflective fiber-optic icing sensor and the transmission fiber-optic icing sensor. However, both of them have some shortcomings, i.e., small measurement range and low measurement accuracy.

This paper presents a new fiber-optic icing sensor with multi-light paths. The optical path of fiber-optic icing sensor is improved. Support vector regression (SVR) is utilized to model the sensor for estimating icy thickness.

\section{BASIC THEORY}

\section{A. The Basic Principle of the Reflective Fiber-Optic Icing Sensor}

The reflection and refraction occurs when the light generated by the transmitting fiber reaches the air-ice interface, the ratio between reflection light flux and incident light flux is called reflectance, which is represented by $\rho$ in equation 1 .

$$
\rho=\left[\left(n^{\prime}-n\right) /\left(n^{\prime}+n\right)\right]^{2}
$$

$n^{\prime}$ is the refractive index of a medium, $n$ is the refractive index of a medium.

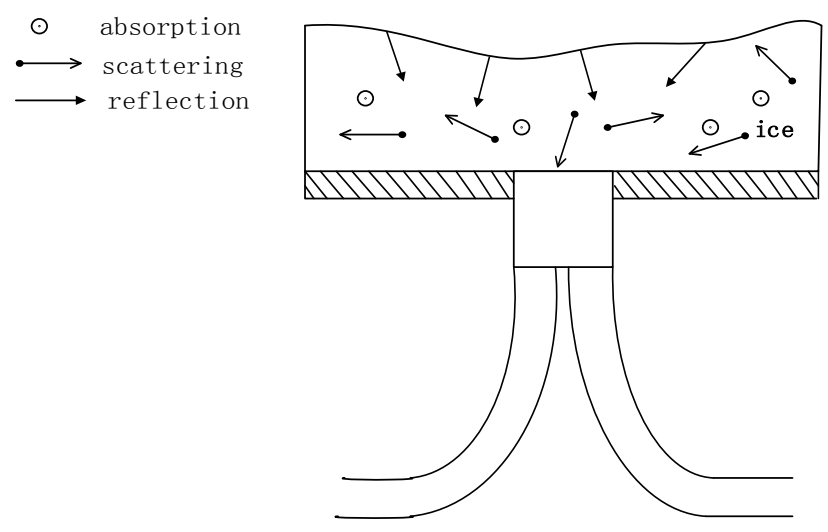

FIGURE I. SCHEMATIC DIAGRAM OF THE REFLECTIVE FIBEROPTIC ICING SENSOR

Equation 1shows that at the interface between the light source and the ice layer, almost all the light entrances into the ice, the loss of the incident light intensity is very small(as is shown in Figure I). The reflected light intensity will reach the maximum corresponding to a certain ice thickness. If the ice thickness increases continuously, the optical fiber probe mainly receives the scattered light. Therefore, the reflected light intensity will reduce Reflected light intensity will reach saturation with the increase of icy thickness which limits range of the ice measurement.

\section{B. The Basic Principle of the Transmission Fiber-Optic Icing} Sensor

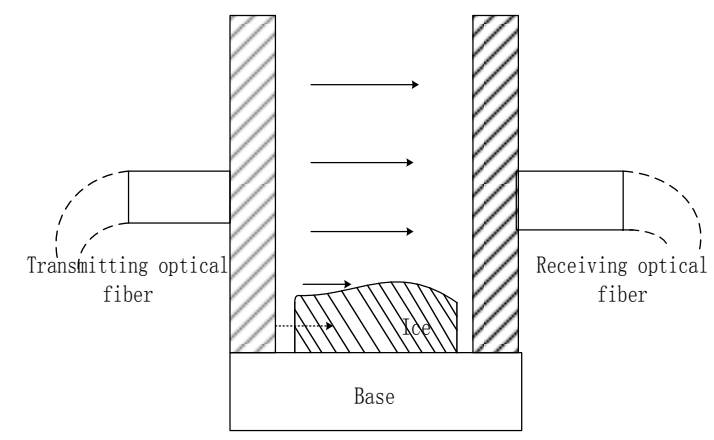

FIGURE II. SCHEMATIC DIAGRAM OF THE TRANSMISSION FIBEROPTIC ICING SENSOR

The principle of the transmission fiber-optic icing sensor is shown in Figure II. All incidents light will reach the detecting 
fiber in condition of no ice. The absorption and reflection of the ice occurs, this will weaken the light intensity detected by optical fiber probe. With the increase of ice thickness, the light intensity received becomes weak gradually. So the ice thickness can be measured according to the light intensity. The basic principle of fiber-optic icing sensor is similar to that of reflective fiber-optic icing sensor.

\section{THE DESIGN OF THE MULTI-LIGHT PATHS FIBER-OPTIC ICING SENSOR}

\section{A. Optical Design}

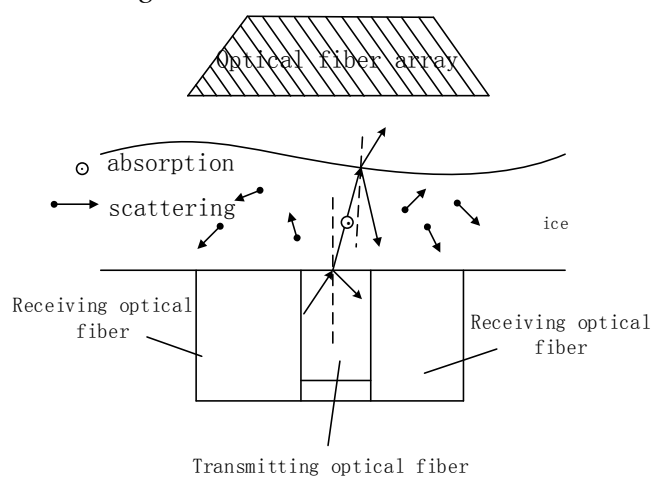

FIGURE III. SCHEMATIC DIAGRAM OF MULTI-LIGHT PATHS FIBEROPTIC ICING SENSOR

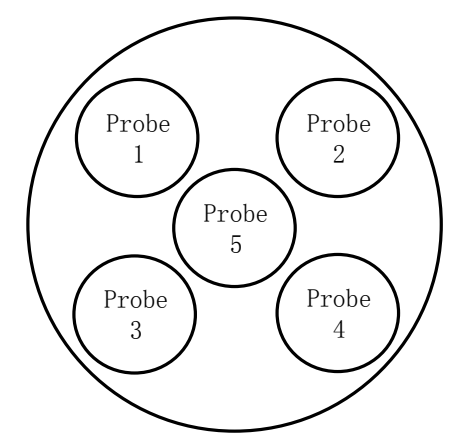

FIGURE IV. SCHEMATIC DIAGRAM OF OPTICAL FIBER ARRAY

In this paper, a new method for measuring the ice thickness is proposed, which integrates the advantages of the reflective fiber-optic icing sensor and the transmission fiber-optic icing sensor. The Figure III shows the optical structure. In this method, a set of fiber array is added to measure the transmission intensity of light. In order to absorb more transmitted light to reduce the interference, the multi-channel array is used. As is shown in Figure IV, there are five channels in the array; the optical fiber probe in the center is coaxial with the transmitting optical fiber. The other four channels are $1 \mathrm{~mm}$ apart from the axis, and they are uniformly distributed in a concentric circle. Light from the bottom of the ice is detected by the reflective optical fiber probe, and light above the ice is detected by the optical fiber array.

\section{B. Hardware Structure}

Figure V shows the hardware structure, the transmitted light and reflected light are detected by the optical fiber probe. The photoelectric detector transforms the light intensity signal to the voltage signal, and then the voltage signal is differentially amplified and low-pass filtered by signal conditioning circuit. The A/D converter converts the analog signals to digital signals. At last, the digital signals are transmitted to the control board through 485 buses for processing, and then the data will be transmitted to the host computer through GPRS network.

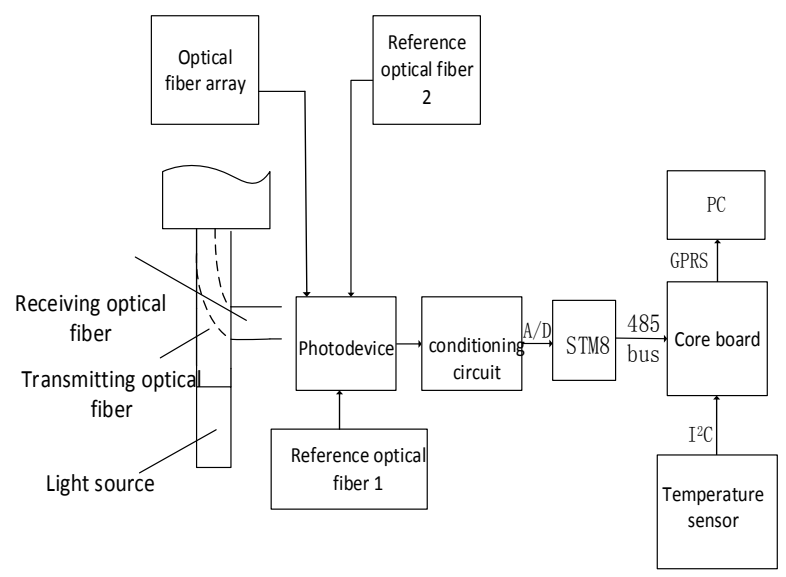

FIGURE V. SYSTEM STRUCTURE DIAGRAM

The reference optical fiber 1 was installed on the same side with the reflective optical fiber. In order to make the reference optical fiber 1 not receive the reflected light, they are kept in a certain distance. Through differential amplification, the output result is related to the ice thickness, which can exclude the influence of the instability of optical path and amplifier noise. Similarly, the reference optical fiber 2 was used to remove the interference of the transmission optical fiber.

At different temperatures, the forms of the ice are different, so the reflection and absorption of light in ice are different. The effects of temperature must be taken into account a temperature detecting module is thus added to the system.

\section{EXPERIMENT AND RESULT ANALYSIS}

\section{A. Sensor Modeling}

SVMs are a class of machine learning algorithms proposed by Vapnik et al. which has excellent learning performance. SVR, a kind of SVMs, is primarily applied to solving regression problems. In this work, modeling icing sensor is essentially to train a SVR.

This Icing sensor model is shown as Figure VI. The input factors are the values of the transmission intensity, the reflective intensity, and the site temperature. The output is ice thickness.

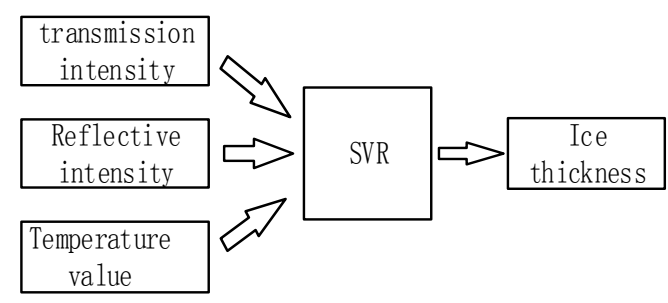

FIGURE VI. SENSOR MODEL BASED ON SVR 
Modeling process of this ice sensor is described in the following. The training samples are obtained through our experiment, and then appropriate kernel function and related parameters are chosen to training the SVR. Finally, using SVR model to estimate ice thickness.

\section{B. Experimental Method And Data Analysis}

In order to illustrate the advantages of the fiber-optic icing sensor with multi-light paths, we obtained experimental data and drew the response curve of the light intensity under the condition of constant temperature and illumination. In order to handle the influence of temperature, the SVR was trained using the samples with different temperature.

In the experiment, the led with $940 \mathrm{~nm}$ wavelength is chosen as the light source, the distance between the transmission optical fiber probe and the reflecting optical fiber probe is $15 \mathrm{~mm}$. The ice thickness is accurately measured by an electron microscope (as is shown in Figure VII.).

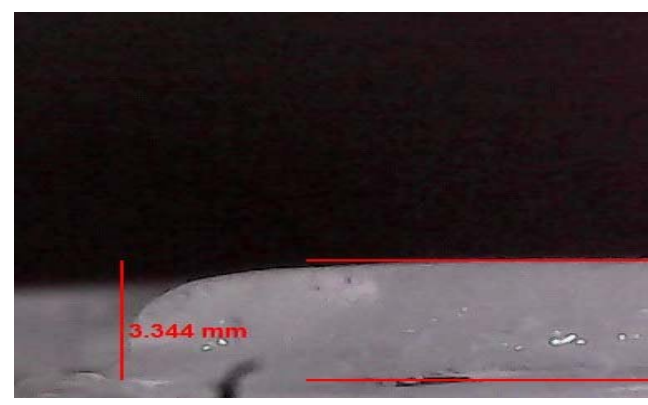

FIGURE VII. CALIBRATION OF THE ICE THICKNESS

Firstly, the light intensity response curve (as shown in figure VIII) was generated according to the experimental data, which is obtained at five below zero. According to the curve, the reflected light intensity reaches the maximum when the ice thickness is $1.48 \mathrm{~mm}$, and it is saturated when the ice thickness is $3.8 \mathrm{~mm}$., so it is hard to forecast the ice thickness if the range of the ice thickness is unknown. At the same time, with the increase of ice thickness, the transmission intensity weakens gradually, it is close to zero when the ice thickness exceeds $8 \mathrm{~mm}$.

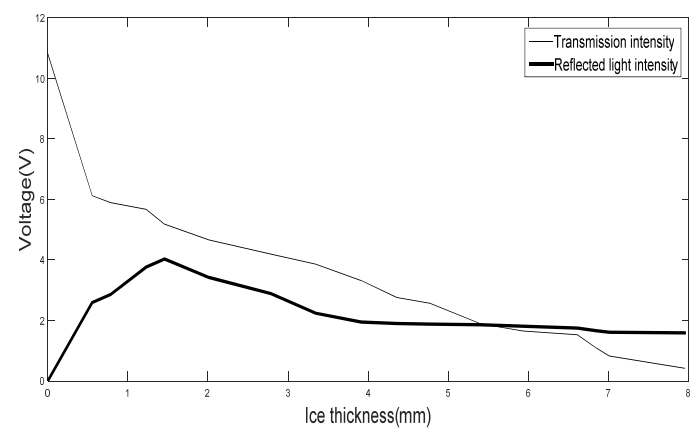

FIGURE VIII. CURVE OF LIGHT INTENSITY AT $-5^{\circ} \mathrm{C}$

Secondly, the influence of temperature is considered in the experiment. The measurement results contain the temperature value. The experimental results are shown in Table I,
TABLE I. EXPERIMENTAL DATA INCLUDING TEMPERATURE VALUE

\begin{tabular}{|c|c|c|c|}
\hline $\mathbf{T}\left({ }^{\circ} \mathrm{C}\right)$ & $\begin{array}{c}\text { Ice } \\
\text { thickness(mm) }\end{array}$ & $\begin{array}{c}\text { Transmission } \\
\text { intensity(V) }\end{array}$ & $\begin{array}{c}\text { Reflective } \\
\text { intensity(V) }\end{array}$ \\
\hline-1.2 & 0 & 10.81 & 0 \\
\hline-1.3 & 0.554 & 6.12 & 2.59 \\
\hline-2.2 & 0.785 & 5.89 & 2.86 \\
\hline-2.4 & 1.227 & 5.27 & 3.76 \\
\hline-2.7 & 1.234 & 5.18 & 4.03 \\
\hline-3.2 & 2.014 & 4.46 & 3.42 \\
\hline-2.4 & 2.786 & 4.09 & 3.11 \\
\hline-2.8 & 3.344 & 3.76 & 2.24 \\
\hline-3.4 & 3.913 & 3.12 & 1.95 \\
\hline-2.5 & 4.357 & 2.66 & 1.90 \\
\hline-1.8 & 4.773 & 2.37 & 1.88 \\
\hline-4 & 5.405 & 1.89 & 1.86 \\
\hline-3.5 & 5.932 & 1.65 & 1.81 \\
\hline-4.1 & 6.613 & 1.33 & 1.75 \\
\hline-2.5 & 6.856 & 1.08 & 1.66 \\
\hline-3.6 & 7.012 & 0.83 & 1.61 \\
\hline-4.7 & 7.952 & 0.42 & 1.59 \\
\hline
\end{tabular}

TABLE II. THE PREDICTION DATA AND THE MEASUREMENT DATA OF THE MODEL

\begin{tabular}{|c|c|c|c|c|c|c|}
\hline NO & 1 & 2 & 3 & 4 & 5 & 6 \\
\hline $\mathbf{T}\left({ }^{\circ} \mathrm{C}\right)$ & -1.2 & -2.3 & -3.4 & -3.6 & -4.1 & -1.8 \\
\hline $\begin{array}{c}\text { Transmission } \\
\text { intensity (V) }\end{array}$ & 6.11 & 4.56 & 2.51 & 2.25 & 1.51 & 0.82 \\
\hline $\begin{array}{c}\text { Reflective } \\
\text { intensity (V) }\end{array}$ & 2.34 & 4.05 & 4.12 & 2.16 & 1.95 & 1.56 \\
\hline $\begin{array}{c}\text { Prediction } \\
\text { Value(mm) }\end{array}$ & 1.271 & 2.094 & 3.914 & 5.101 & 5.660 & 6.217 \\
\hline $\begin{array}{c}\text { Measurement } \\
\text { value(mm) }\end{array}$ & 1.324 & 2.181 & 3.827 & 5.211 & 5.804 & 6.362 \\
\hline $\begin{array}{c}\text { Relative error } \\
\text { rate (\%) }\end{array}$ & 4 & 3.9 & 2.3 & 2.1 & 2.4 & 2.3 \\
\hline
\end{tabular}

The SVR is used to model correlation among temperature, transmission intensity, reflected light intensity and ice thickness. Six samples are chosen to verify the model. The real value and the estimation value of the samples are shown in Table II. The error rate curve of the model is shown in Figure IX. It can be seen from the graph that the relative error rate of the model is about 3 percent. The estimation accuracy is very high when the ice thickness is within $7 \mathrm{~mm}$. Our method can both expand measuring range and improve the measurement accuracy.

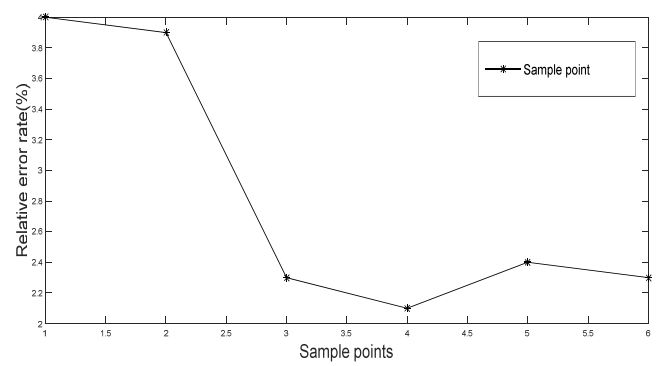

FIGURE IX. RELATIVE ERROR RATE OF THE MODEL

\section{CONCLUSION}

In allusion to the drawbacks of fiber-optic icing sensor, this paper presents a novel icing sensor method. Based on the 
reflective fiber-optic icing sensor, a set of fiber array is added to measure the transmission intensity of the light, and the SVR is used to model this sensor. The experimental results show that in comparison to reflective fiber-optic icing sensor, this developed sensor has a larger measurement range, higher accuracy and stronger anti-interference ability.

\section{REFERENCES}

[1] Li Wei, Ye Lin ,Zhang Jie, Zhang Hong. Experimental study on the fiber-optic sensor for direct ice detection [J]. J.Huazhon Univ.of Sci. \&Tech.(Natural Science Edition), 2009(08).

[2] Cong Hong.,Reflective optical fiber micro-displacement sensor[J].Journal of Transducer Technology, 2003(09).

[3] Wang Hua, Wang Yilun, Zhang Binhua. Theory of Designing Ice Detector Base On Magnetostriction[J]. Transactions of China Electrot Echnical Society, 2003(06).

[4] Zhang Jie, Zhou Lei, Zhang Hong, Ye Lin. Aircraft icing detection technology[J]. Chinese Journal of Scientific Instrument, 2006(12).

[5] Wang Lin, Li Hengwei, Wang Xuewei. Optical fiber sensor and its application lecture (six), sixth stresses ,displacement optical fiber sensor[J]. Electrical Measurement \& Instrumentation, 1996(08).

[6] Yang Huayong,Lu Haibao,Xu Tao. Summary on the Reflective Intensity-modulated Fiber Optic Sensor[J],Journal of sensing technology,2001(04).

[7] MAKKONEN L.Modeling of Ice Accretion on Wires. Journal of Applied Meteorology . 1984

[8] PRUZAN D A,KHATKHATE A A.Capacitive Ice Detector Development. 1993

[9] kiades A A.Measurement of optical diffusion prop-erties of ice for direct detection ice accretion Sensors. Sensors and Actuators . 2007 